\title{
CLINICAL PROFILE AND ROLE OF SEPTIC SCREEN IN EARLY ONSET SEPSIS IN A TERTIARY CARE HOSPITAL OF WESTERN NEPAL-A PROSPECTIVE OBSERVATIONAL STUDY
}

Binod Kumar Gupta, ${ }^{1}$ Raju Kaphle, ${ }^{1}$ Buby Philip Kurian, ${ }^{1}$ Badri Kumar Gupta ${ }^{1}$

\section{ABSTRACT}

\section{INTRODUCTION}

Neonatal sepsis is an important cause of neonatal mortality and morbidity with wide range of clinical manifestations. This study was aimed to study the clinical characteristics of sepsis along with the role of septic screen for early diagnosis of septicemia.

\section{MATERIAL AND METHODS}

A prospective observational hospital-based cross-sectional study was conducted in 113 screen positive newborns over a 12-month period at Universal College of Medical Sciences, Teaching Hospital, Bhairahawa, Nepal.

\section{RESULTS}

Out of 489 cases admitted to the NICU during the study period, 113 babies with screen-positive sepsis were included in the study. Poor feeding $(46 \%, n=52)$, respiratory distress $(38.9 \%, n=44)$ and lethargy $(30.1 \%, n=34)$ were top three clinical presentations in neonates with sepsis followed by seizures, jaundice, vomiting, fever, and hypothermia respectively. $57.5 \%(n=65)$ of clinical sepsis cases enrolled had culture positivity with Staphylococcus aureus in $41.5 \%(n=27)$ and coagulase negative Staphylococcus (CONS) in $27.7 \%(\mathrm{n}=18)$. Klebsiella was the third common organism isolated in blood culture $(23.1 \%, \mathrm{n}=15)$.

The sensitivities and specificities of two-test and three-test combinations in proven sepsis was calculated. Two-test combination showed sensitivities between $33-100 \%$ and specificities between $30-90 \%$ whereas three-test combination showed the sensitivities and specificities between $60-100 \%$ and $20-90 \%$ respectively.

\section{CONCLUSION}

Poor feeding, respiratory distress and lethargy were common presentations in early-onset neonatal sepsis. Three-test combination of septic screen had no overall advantage over two-test combination in the present study.

KEYWORDS Neonatal sepsis, Septic screen, CRP

1. Department of Pediatrics, Universal College of Medical Sciences, Bhairahawa, Nepal

DOI: http//doi.org/10.3126/jucms.v8i1.29826

For Correspondence

Dr. Binod Kumar Gupta

Department of Pediatrics

Universal College of Medical Sciences,

Bhairahawa, Nepal

Email:drbinodkgupta@hotmail.com 


\section{INTRODUCTION}

Neonatal sepsis is a significant cause of neonatal morbidity and mortality in the newborn. ${ }^{1}$ Globally, the estimated neonatal morbidity and mortality cases range between 2.53 million annually. ${ }^{2}$ It is estimated that $20 \%$ of all neonates develop sepsis and it is responsible for $30-50 \%$ of total neonatal deaths in developing countries. ${ }^{3}$ In Nepal, septicemia and emergence of drug resistant bacteria have been found to be important predictors of neonatal mortality. According to Nepal Demographic and Health Survey (2011), 85\% of total death is accounted to neonatal sepsis which is higher than the previous surveys, $70 \%$ in 2006 and $69 \%$ in $2001 .{ }^{4} \mathrm{NMR}$ is higher in rural areas (34 deaths per 1000 live-births) than in urban areas (23 deaths per 1000 live births).

The fate of a neonate with sepsis largely depends on early detection and treatment accordingly. The diagnosis of neonatal sepsis may not be easy clinically. Many risk factors are associated with neonatal sepsis, and clinical features of neonatal sepsis are often non-specific. Though blood culture is the gold standard in the diagnosis of neonatal sepsis, it is not always positive even if clinical features of sepsis are present in the neonates. Also, a positive blood culture report is only obtained in $25-40 \%$ of cases and requires a period of about 48 72 hours. ${ }^{3}$ Therefore, high index of suspicion is necessary for early diagnosis of sepsis, and when blood culture tests are negative. In these conditions, a combination of septic screen tests may help in the diagnosis. In developing countries like Nepal where the culture facilities are not available in many district hospitals, it becomes more essential to consider septic screening tests.

Hence, there is a role of sepsis screen (a battery of rapid diagnostic test) for early diagnosis of septicemia and identification of culture negative cases. This study was, therefore, conducted to study the clinical profile of septic babies along with the role of septic screen for early diagnosis of septicemia to identify culture negative sepsis and compare rapid diagnostic tests with blood cultures.

\section{MATERIAL AND METHODS}

A hospital-based prospective observational study was carried out in Neonatal ward at Universal College of Medical Sciences, Bhairahawa, Nepal from July 2013 to July 2014. The study was approved by the Institute's ethics committee. All inborn and out born neonates of either sex from birth to 72 hours of life having clinical symptoms of sepsis and maternal risk factors such as (prolonged rupture of membranes, fever, meconium stained liquor, prolonged duration of labor, chrioamnionitis) were screened and neonates with screen positive sepsis were included in the study. Babies who received antibiotics prior to the enrollment and patients not willing to give consent were excluded from the study. Sample size of 113 babies was calculated assuming absolute precision of $5 \%$ corresponding to a confidence interval of $50 \% \pm 5 \%$ ) at type 1 error of $5 \%$. Prevalence rate of $8 \%$ was considered for the sample size calculation which was obtained from a study done by Nepal et al. ${ }^{6}$

Blood investigations were sent from all neonates with clinically suspected sepsis. Total leukocyte count (TLC), differential leukocyte count (DLC), immature to total neutrophil ratio (I/T ratio), peripheral smear for band cells and toxic granules,C-reactive protein (CRP) by latex agglutination technique and $\mu$ ESR using standardized nonheparinized capillaries were sent. Blood culture was sent after obtaining blood samples (approximately $2 \mathrm{ml}$ ) from peripheral vein after proper cleaning with iodine and spirit. Chest $\mathrm{x}$-ray was considered whenever necessary. The cut-off values for positive tests were TLC $<5000 / \mathrm{mm}^{3}$ or $>20000$ $/ \mathrm{mm}^{3}, \mathrm{I} / \mathrm{T}$ ratio $\geq 0.20, \mathrm{CRP}$ positive or negative and $\mu \mathrm{ESR}$ $\geq 15 \mathrm{~mm}$ in first hour. Clinical sepsis was considered in babies with two or more parameters positive for septic screen also called "screen positive sepsis".

Data was entered into excel sheet and analysis was done by using SPSS 21 software. All screen positive sepsis were subjected to analysis with comparison to culture positive cases by application of appropriate statistical tools. Descriptive analysis was done using mean and percentage as statistical tools. Sensitivity, specificity, positive predictive value (PPV) and negative predictive value (NPV) were calculated for the septic screen parameters. Sensitivities and specificities were also calculated for two-test and three-test combinations.

\section{RESULTS}

Out of 489 cases admitted to the NICU during the study period, 113 babies had screen positive sepsis and were included in the study. Out of 113 screen positive babies included in the study, the proportion of male babies were more than female babies $(n=77,68.1 \%$ versus $n=36,31.9 \%)$. Majority of babies ( $\mathrm{n}=83,73.5 \%)$ were born by lower segment cesarean section (LSCS). 45.1\% $(n=51)$ were low birth weight $(<2500 \mathrm{gm})$ and $31 \%(\mathrm{n}=35)$ were preterm. About $62.8 \%$ of mothers were in between $20-25$ years with $23.9 \%$ in between 26-30 years and $9.7 \%$ above $>30$ years of age. Only $3.5 \%$ mothers were less than $<20$ years.

Table 1 presents the baseline characteristics of maternal distribution according to clinical examination. Fever was only present in $6.2 \%(\mathrm{n}=7)$ mothers. Per vaginal examination more than 3 times was found to be done in $57.5 \%(n=65)$ of mothers. $20.4 \%(n=23)$ mothers had meconium stained liquor (Table 1$)$ 
Table 1. Maternal distribution according to clinical examination

\begin{tabular}{lcc}
\hline Clinical examination & $\begin{array}{c}\text { Number } \\
(\mathbf{n}=\mathbf{1 1 3})\end{array}$ & $\begin{array}{c}\text { Percentage } \\
(\mathbf{\%})\end{array}$ \\
\hline Fever & 7 & 6.2 \\
Per vaginal examination $>3$ times & 65 & 57.5 \\
FHR $>160$ bpm & 2 & 1.8 \\
Duration of PROM $>12$ hours & 30 & 26.5 \\
Meconium stained liquor & 23 & 20.4 \\
Duration of labor $>24$ hours & 11 & 9.7 \\
\hline
\end{tabular}

bpm- beats per minute, PROM- prolonged rupture of membranes

CRP was positive among 50.4\% neonates and toxic granules present in peripheral smear werefound in $37.2 \%$ of neonates. The mean values of $\mathrm{Hb}$, TLC, immature/total neutrophil, micro ESR (mm) was found as shown in the table (Table 2).

\section{Table 2. Biochemical parameters of enrolled neonates}

\begin{tabular}{ll}
\hline $\begin{array}{l}\text { Biochemical } \\
\text { parameters }\end{array}$ & \multicolumn{1}{c}{$\begin{array}{c}\text { Mean } \pm \text { SD } \\
(\mathbf{n}=113)\end{array}$} \\
\hline $\mathrm{Hb}(\mathrm{gm} / \mathrm{dl})$ & $16.49 \pm 2.32$ \\
$\mathrm{TLC}\left(\mathrm{cells} / \mathrm{mm}^{3}\right)$ & $17960.65 \pm 9745.55$ \\
$\begin{array}{l}\text { Immature/Total neutrophil } \\
\text { ratio ( } / \text { T ratio) }\end{array}$ & $0.22 \pm 0.10$ \\
$\mu \mathrm{ESR}\left(\mathrm{mm}\right.$ in $1^{\text {st }}$ hour $)$ & $11.27 \pm 5.49$ \\
\hline
\end{tabular}

Poor feeding $(46 \%, \mathrm{n}=52)$, respiratory distress $(38.9 \%, \mathrm{n}=44)$ and lethargy $(30.1 \%, \mathrm{n}=34)$ were top three clinical presentations in neonates with sepsis followed by seizures, jaundice, vomiting, fever and hypothermia respectively (figure 3).

\section{Table 3. Clinical profile of septic babies $(n=113)$}

\begin{tabular}{lcc}
\hline Clinical Features & Number $(\mathbf{N})$ & Percentage (\%) \\
\hline Poor feeding & 52 & 46 \\
Fever & 14 & 12.4 \\
Lethargy & 34 & 30.1 \\
Seizures & 26 & 23 \\
Jaundice & 23 & 20.4 \\
Vomiting & 16 & 14.2 \\
Respiratory distress & 44 & 38.9 \\
Hypothermia & 4 & 3.5 \\
Poor cry & 41 & 36.3 \\
Diminished activity & 34 & 30.1 \\
\hline
\end{tabular}

The sensitivity and specificity of CRP in detecting culture positive neonatal sepsis was found to be $64.6 \%$ and $68.8 \%$ with positive predictive value (PPV) and negative predictive value (NPV) of $73.7 \%$ and $58.9 \%$ respectively. The accuracy of CRP was $66.3 \%$. The sensitivity, specificity, PPV, NPV and accuracy of micro-ESR were $67.7 \%, 81.2 \%, 83 \%, 65 \%$ and $73 \%$ respectively whereas of I/T ratio were $60 \%, 68.7 \%, 72 \%$,
$55 \%$, and $63.7 \%$ respectively as shown in Table 4 .

The sensitivity and specificity was calculated combining two or three septic screen parameters. Two-test combination viz $\mathrm{CRP}+\mathrm{TLC}, \mathrm{CRP}+\mathrm{I} / \mathrm{T}$ ratio, $\mathrm{TLC}+\mathrm{I} / \mathrm{T}$ ratio $\mathrm{TLC}+\mathrm{ANC}$, $\mathrm{TLC}+\mu \mathrm{ESR}, \mathrm{I} / \mathrm{T}$ ratio $+\mathrm{ANC}, \mathrm{I} / \mathrm{t}$ ratio $+\mu \mathrm{ESR}, \mathrm{ANC}+\mu \mathrm{ESR}$ showed sensitivities and specificities of $100 \%$ and $40 \%, 73 \%$ and $90 \%, 80 \%$ and $80 \%, 67 \%$ and $90 \%, 100 \%$ and $70 \%, 73 \%$ and $90 \%, 100 \%$ and $30 \%, 93 \%$, and $40 \%$ respectively whereas three-test combination viz $\mathrm{TLC}+\mathrm{I} / \mathrm{T}$ ratio $+\mathrm{ANC}, \mathrm{TLC}+\mathrm{I} / \mathrm{t}$ ratio $+\mathrm{CRP}, \mathrm{TLC}+\mathrm{I} / \mathrm{T}$ ratio $+\mathrm{ANC}, \mathrm{TLC}+\mathrm{ANC}+\mathrm{CRP}$, $\mathrm{TLC}+\mathrm{ANC}+\mu \mathrm{ESR}+\mathrm{TLC}+\mathrm{CRP}+\mu \mathrm{ESR}, \mathrm{I} / \mathrm{T}$ ratio, $\mathrm{ANC}+\mathrm{CRP}$, $\mathrm{I} / \mathrm{T}$ ratio $+\mathrm{NC}+\mu \mathrm{ESR}, \mathrm{I} / \mathrm{T}$ ratio $+\mathrm{CRP}+\mathrm{ANC}$ showed the sensitivities and specificities of $60 \%$ and $80 \%, 80 \%$ and $80 \%$, $100 \%$ and $20 \%, 87 \%$ and $70 \%, 73 \%$ and $30 \%, 73 \%$ and $90 \%$, $100 \%$ and $30 \%, 100 \%$ and $30 \%, 80 \%$, and $90 \%$ respectively.

Table 4. Sensitivity, specificity, PPV, NPV and accuracy of septic screen parameters

\begin{tabular}{lccccccc}
\hline $\begin{array}{l}\text { Septic Screen } \\
\text { parameters }\end{array}$ & \multicolumn{2}{c}{ Culture } & Sensitivity & Specificity & PPV & NPV & Accuracy \\
\hline CRP & Positive & Negative & & & & & \\
Positive & 42 & 15 & 64.6 & 68.8 & 73.7 & 58.6 & 66.3 \\
Negative & 23 & 33 & & & & & \\
$\mu$ ESR & & & & & & & \\
Positive & 44 & 9 & 67.7 & 81.2 & 83 & 65 & 73 \\
Negative & 21 & 39 & & & & & \\
I/T ratio & & & & & & & \\
Positive & 9 & 15 & 60 & 68.7 & 72 & 55 & 63.7 \\
Negative & 26 & 33 & & & & & \\
\hline
\end{tabular}

$57.5 \%(n=65)$ of clinical sepsis cases enrolled had culture positivity with Staphylococcus aureus in $41.5 \%(\mathrm{n}=27)$ and coagulase negative Staphylococcus (CONS) in $27.7 \%(\mathrm{n}=18)$. Klebsiella was the third common organism isolated in blood culture $(23.1 \%, \mathrm{n}=15)$ (Table 5$)$.

\section{Table 5. Bacteriological profile of organisms isolated}

\begin{tabular}{lcc}
\hline Blood culture & Number (N=113) & Percentage (\%) \\
Positive & 65 & 57.5 \\
Negative & 48 & 42.5 \\
\hline Isolates detected & $(\mathrm{n}=65)$ & \\
\hline Citrobacter & 1 & 1.5 \\
Coagulase negative & 18 & 27.7 \\
Staphylococcus (CONS) & 3 & 4.6 \\
E. coli & 15 & 23.1 \\
Klebsiella & 1 & 1.5 \\
Proteus mirabilis & 27 & 41.5 \\
Staphylococcus aureus & & \\
\hline
\end{tabular}




\section{DISCUSSION}

Neonatal sepsis is an important cause of neonatal mortality and morbidity in low and middle incomes countries like Nepal. It is extremely important to make an early diagnosis of sepsis, because early institution of empirical antimicrobial therapy may be life saving. ${ }^{7}$

Out of 489 babies admitted in the NICU during the study period, $23 \%(n=113)$ had screen positive early onset sepsis. A retrospective study conducted by Shrestha et al in Nepal found $20 \%$ cases to be culture positive (103 cases culture positive out of 513 screen positive). ${ }^{1}$ In contrast to his study, the present study had culture positivity in $57.5 \%(n=65)$ cases which was higher than the study conducted by him. A study done by Lakhey et al found culture positivity of $66.3 \%(n=65)$ out of total 98 screen positive newborns which was even more than the present study. They also concluded that $90.35 \%$ of total culture positive sepsis had screen positive sepsis. ${ }^{8}$ Another study done at a tertiary care hospital of Nepal showed culture positivity rate of $6.1 \%$ (54 cases culture positive out of 1349 cases. ${ }^{9}$ The high incidence of culture positivity in the present cases could be due to inclusion of only screen positive cases whereas the low incidence of culture positivity in some studies were most probably due to inclusion of all suspect cases of clinical cases in its calculation.

The clinical features of early onset neonatal sepsis are often subtle and non-specific which necessitates the high index of suspicion. Poor feeding $(46 \%, \mathrm{n}=52)$, respiratory distress $(38.9 \%, n=44)$ and lethargy $(30.1 \%, n=34)$ were top three clinical presentations in neonates in the present study followed by seizures, jaundice, vomiting, fever and hypothermia respectively. A study conducted by Khinchi et al (2010) also concluded refusal to feed $(74 \%, n=160)$ and respiratory distress $(75 \%, \mathrm{n}=161)$ being two major clinical presentations in neonates with sepsis. In their study, fever was present in $69 \%(n=148)$ cases which was higher than in the present study. ${ }^{10}$ A recent study done in Nepal also concluded respiratory distress $(56 \%, \mathrm{n}=84)$, fever $(26 \%, \mathrm{n}=39)$ and feeding difficulty $(12.7 \%, \mathrm{n}=19)$ being the major manifestations of sepsis in newborn. ${ }^{8}$ A study conducted in a tertiary care hospital in Bangladesh concluded fever (44.4\%), respiratory distress $(27.8 \%)$ and poor feeding $(22.2 \%)$ to be the major manifestations of neonatal sepsis. In the same study, they documented hypothermia in $11.1 \%$, apnea in $16.7 \%$, cyanosis in $11.1 \%$, convulsions in $11.1 \%$ and jaundice in $50 \%$. ${ }^{11}$ This variation in clinical features of neonatal sepsis may probably be due to different influencing factors like birth weight, delivery site, age of the newborn, and referral timing.

In the present study, $57.5 \%(\mathrm{n}=65)$ neonates of early onset sepsis had culture positivity where Staphylococcus aureus
$(41.5 \%, \mathrm{n}=27)$, coagulase negative Staphylococcus $(27.7 \%$, $\mathrm{n}=18)$ and Klebsiella $(23.1 \%, \mathrm{n}=15)$ were three predominant isolated organisms. Lakhey et al (2015-2016) in their study, also found similar proportions of Staphylococcus aureus isolated in newborns $(41.7 \%, \mathrm{n}=30){ }^{8}$

The sensitivity, specificity, PPV and NPV of CRP in the present study were $64.6 \%, 68.8 \%, 73.7 \%$, and $58.6 \%$. In a study done by Lakhey et $\mathrm{al}^{8}$, these parameters were comparatively more than the present study (sensitivity$77.8 \%$, specificity-66.7\%, PPV-68.2\%, and NPV- 76.5\%). Bhale et $\mathrm{al}^{12}$ found that these parameters were even more (sensitivity-84.62\%, spscificity-78\%, PPV-77.78 and NPV84.78 ) than the study done by Lakhey et al and the present study. In contrast, Swarnakar et al (2012) found the sensitivity, specificity, PPV and NPV of $52.3 \%, 56 \%, 89 \%$ and $14.3 \%$ respectively which were less than the present study and the previous studies. ${ }^{13}$

The sensitivity, specificity, PPV and NPV of $\mu$ ESR in the present study were $67.7 \%, 81.2 \%, 83 \%$, and $65 \%$ which was almost similar as in the study conducted by Bhale et al. ${ }^{12}$ In contrast, these parameters were less in a study conducted by Lakhey at al (sensitivity-51.4\%, specificity- 60.2\%, PPV$54 \%$, and NPV- $57.3 \%)^{8}$

The sensitivity, specificity, PPV, and NPV of I/T ratio in the present study were $60 \%, 68.7 \%, 72 \%$, and $55 \%$ respectively. The findings were almost comparable in a study conducted by Lakhey et al (sensitivity- 73\%, specificity- 61.5\%, PPV$63.8 \%$ and NPV- $71.6 \%) .{ }^{8}$ Jadhav et al $(2013)^{14}$ and Bhale et al $(2015)^{12}$ found the sensitivity of $\mathrm{I} / \mathrm{T}$ ratio $(80 \%$ and $75.82 \%$ respectively) to be more than the present study whereas the specificity was less ( $65 \%$ and $66.35 \%$ respectively).

Lakhey at $\mathrm{al}^{8}$ found the sensitivity and specificity in two-test combination to be $90.3 \%$ and $75.6 \%$ whereas it was $100 \%$ and $83 \%$ respectively in a study done by Chirico et al. ${ }^{15}$ On two-test combination, the sensitivities in the present study ranged from $33 \%-100 \%$ with specificities in between $30 \%-90 \%$. Threetest combination revealed sensitivities and specificities in between $60 \%-100 \%$ and $20 \%-100 \%$. We could not find any added benefit in diagnosing sepsis on three-test combination in comparison to the two-test combination.

This study had few limitations. Firstly, we only included early- onset sepsis cases in the present study. Inclusion of lateonset sepsis cases along with the early onset septic cases would have given a clearer picture on the clinical profile and laboratory findings of septic newborns. Secondly, this was a hospital-based study which may not give a true picture of septic cases in the community. Therefore, multicentric trials with large sample size should be considered. 


\section{CONCLUSION}

Poor feeding, respiratory distress and lethargy were top three clinical presentations in neonates. Combining two or more parameters increased the sensitivity and specificity of the septic screen in comparison to the individual parameter. Three-test combination did not reveal any additional benefit in comparison to two-test combination in the diagnosis of sepsis.

\section{CONFLICT OF INTERESTS}

None.

\section{AUTHOR CONTRIBUTIONS}

BKG (Binod Kumar Gupta) conceptualized the study, performed the literature search, analyzed the data, drafted the manuscript, revised it and supervised the study. RK performed the literature search, analyzed the data and drafted the manuscript. BPK collected the data and helped in analysis, drafting and revision of the manuscript. BKG (Badri Kumar Gupta) drafted the manuscript, revised it and supervised the study.

\section{ACKNOWLEDGEMENTS}

The authors thank Dr. Nagendra Chaudhary, Department of Pediatrics, UCMS, Nepal for his technical support and guidance during the manuscript preparation.

\section{REFERENCES}

1. Shrestha P, Das BK, Bhatta NK, Jha DK, Das B, Setia A, Tiwari A. Clinical and Bacteriological Profiles of Blood Culture Positive Sepsis in Newborns. J Nepal Paedtr Soc. 2007; 27(2):64-7.

2. Darmstadt GL, Zaidi AK, Stoll BJ. Neonatal infections: a global perspective. InInfectious Diseases of the Fetus and Newborn 2011 Jan 1 (pp. 24-51). WB Saunders.

3. Stoll BJ. Infections of the neonatal infant. In: Behrman RE, Kliegman RM, editors. Nelson textbook of pediatrics, 18th edition. W.B. Saunders Company, 2008, p.794-98.

4. Ministry of Health and Population (MOHP) [Nepal], New ERA, and ICF International Inc. Nepal Demographic and Health Survey. Kathmandu: Ministry of Health and Population, New ERA, and ICF International; 2011.p. 2012.

5. Basavaraj P, Jyothi P, Basavaraj M. Bacteriological profile of neonatal septicemia and antibiotic susceptibility pattern of the isolates. J Nat Sc Biol Med. 2013;4(2):306.

6. Nepal HP, Acharya A, Gautam R, Shrestha S, Paudel R. Bacteriological profile of neonatal septicemia cases and the antimicrobial resistance pattern in a tertiary care hospital of central Nepal. Internat J Biomed Research. 2013;4:26-31.
7. Sankar MJ, Agarwal R, Deorari AK, Paul VK. Sepsis in the newborn. Indian J Pediatr. 2008 Mar;75(3):261-6.

8. Lakhey A, Shakya H. Role of Sepsis Screening in early diagnosis of Neonatal Sepsis. J Pathol Nep. 2017 Mar 30;7(1): 1103-10.

9. Shrestha N, Subedi K, Rai G. Bacteriological Profile of Neonatal Sepsis: A Hospital Based Study. J Nepal Paedtr Soc. 1970 Jan 1;31(1):1-5.

10. Khinchi Y, Kumar A, Yadav S. Profile of Neonatal sepsis. J Coll Med Sci-Nepal. 1970 Jan 1;6(2):1-6.

11. Ahmed ASMNU, Chowdhury MAKA, Hoque M, Darmstadt GL. Clinical and bacteriological profile of neonatal septicemia in a tertiary level pediatric hospital in Bangladesh. Indian Pediatr. 2002 Nov;39(11):1034-9.

12. Bhale C, Kale A, Kale S, Mahajan M, Smulay S. Utility of Sepsis Screen in the Early Diagnosis of Neonatal Sepsis. IJNMR. 2016;4:1001-7.

13. Swarnkar K, Swarnkar M. A study of early onset neonatal sepsis with special reference to sepsis screening parameters in a tertiary care centre of rural India. The Internet Journal of Infectious Diseases. 2012;10(1):36-42.

14. Jadhav S, Misra R, Vyawahare C, Angadi K, Gandham N, Ghosh P. Role of sepsis screen in the diagnosis of neonatal sepsis. Med J DY Patil Univ. 2013;6(3):254.

15. Chirico G, Loda C. Laboratory aid to the diagnosis and therapy of infection in the neonate. Pediatr Rep. 2011 Feb 24;3(1):e1. 